Article

\title{
Essential Oils with High Activity Against Stationary Phase Bartonella Henselae
}

\author{
Xiao Ma ${ }^{1}$, Wanliang Shi ${ }^{1}$ and Ying Zhang ${ }^{1, *}$ \\ 1 Department of Molecular Microbiology and Immunology, Bloomberg School of \\ Public Health, Johns Hopkins University, Baltimore, MD 21205, USA; xma37@jhmi.edu (X.M.); wshi3@jhu.edu \\ (W.S.) \\ * Correspondence: yzhang@jhsph.edu; Tel.: +1-410-614-2975
}

\begin{abstract}
Bartonella henselae is a fastidious Gram-negative intracellular bacterium which can cause cat scratch disease, endocarditis in humans and animals as well as other complications, leading to acute or chronic infections. The current treatment for Bartonella infections is not very effective due to antibiotic resistance and also persistence. To develop better therapies for persistent and chronic Bartonella infections, in this study, with the help of SYBR Green I/PI viability assay, we performed a high-throughput screening of an essential oil library against stationary phase $B$. henselae. We successfully identified 32 essential oils that had high activity, including four essential oils extracted from Citrus plants, three from Origanum, three from Cinnamomum, two from Pelargonium and two from Melaleuca, as well as frankincense, ylang ylang, fir needle, mountain savory (winter), citronella, spearmint, elemi, vetiver, clove bud, allspice and cedarwood essential oils. The minimal inhibitory concentration (MIC) determination of these 32 top hits indicated they were not only active against stationary phase non-growing $B$. henselae but also had good activity against log phase growing $B$. henselae. The time-kill curve by drug exposure assay showed 13 active hits, including essential oils of oregano, cinnamon bark, mountain savory (winter), cinnamon leaf, geranium, clove bud, allspice, geranium bourbon, ylang ylang, citronella, elemi and vetiver, could eradicate all stationary phase $B$. henselae cells within 7 days at the concentration of $0.032 \%(v / v)$. Two active ingredients, carvacrol and cinnamaldehyde, of oregano and cinnamon bark essential oils, respectively, were shown to be very active against stationary phase $B$. henselae such that they were able to eradicate all the bacterial cells even at the concentration $\leq 0.01 \%(\mathrm{v} / \mathrm{v})$. Our finding of active essential oils may help to develop more effective treatments for persistent Bartonella infections.
\end{abstract}

Keywords: essential oils; Bartonella henselae; persisters; stationary phase; antimicrobial activity

\section{Introduction}

Bartonella species are fastidious, Gram-negative, facultative intracellular pathogens [1-3] that can be transmitted to humans or animals by several arthropod vectors including fleas, sheep keds, lice, sand flies, ticks and potentially mites and spiders. Bartonella bacteria can infect healthy people while considered especially important as opportunistic pathogens [4]. At least thirteen Bartonella species are known to be able to infect humans, causing either acute or chronic infections which could lead to cat scratch disease, endocarditis, bacillary angiomatosis [3], bacteremia and central nervous system pathologies [5]. And this pathogenicity is partly due to their unique infection cycle including the lymphatic stage [6] and intraerythrocytic stage [7,8]. It is laborious using classical bacteriological methods to isolate and culture Bartonella spp. in liquid media especially from clinical samples, which requires specific conditions and prolonged incubation periods [9,10]. Therefore, serology and real-time PCR are often used instead of culture to confirm diagnosis for rapid Bartonella detection clinically $[9,10]$. The first-line antibiotics for treating bartonellosis include doxycycline, 
erythromycin, gentamicin, rifampin, azithromycin, ciprofloxacin as well as some drug combinations like doxycycline plus gentamicin, doxycycline plus rifampin [11,12]. However, there is no single treatment effective for systemic $B$. henselae infections, and antibiotic therapy exhibited poor activity against typical uncomplicated cat scratch disease [11,12]. Therefore, bartonellosis treatment remains a significant problem and without treatment, it could cause high mortality in some patients. The phenomenon of bacterial persistence may partly contribute to the difficulty to treat the disease, because persister bacteria are hard to be eradicated and remain in the host which can revert to growing forms under appropriate conditions and lead to relapse or prolonged infections with symptoms [13]. Thus, identifying drugs that target Bartonella persister cells in stationary phase could provide a promising strategy for developing more effective treatment for bartonellosis. In our recent study, we have identified some promising drug candidates from the FDA drug library that are more active than the current drugs used to treat Bartonella infections. However, their utility remains to be further validated.

Essential oils, also known as volatile oil or ethereal oil, are a concentrated hydrophobic liquid containing volatile chemical compounds extracted from plants. It has many uses in aromatherapy [14], food processing [15] and also the potential in medical therapy [16] especially with recent concerns about antibiotic resistance. Previous in vitro studies have found certain essential oils had antibacterial activity against multidrug resistant Gram-negative clinical isolates [17]. And the rapid high-throughput drug screens using SYBR Green I/PI viability assay [18] have successfully identified many essential oils with high activity against stationary phase Borrelia burgdorferi [19,20], as a surrogate model of persister bacteria [21]. In this study, we adapted the same SYBR Green I/PI methodology to perform an efficient screen using our essential oil collection against stationary phase B. henselae and identified a significant number of essential oils that had better activity against non-growing $B$. henselae cells than most clinically used antibiotics for treating bartonellosis. The implication of the identified active hits for improved treatment of persistent Bartonella infections is discussed.

\section{Results}

2.1. Subsection Screening essential oil collection to identify drugs active against non-growing stationary phase B. henselae

Previously, we have developed a SYBR Green I/PI viability assay for rapid viability assessment of $B$. henselae and have successfully used this assay for high-throughput drug screens against non-growing stationary phase $B$. henselae using FDA drug library [22]. Here we adapted this SYBR Green I/PI viability assay for essential oil screens against $B$. henselae. As described above, a 5-day-old stationary phase $B$. henselae JK53 culture was used to identify active essential oils against stationary phase B. henselae. All 149 essential oils were applied at two concentrations, $0.5 \%(\mathrm{v} / \mathrm{v})$ and $0.25 \%(\mathrm{v} / \mathrm{v})$, respectively, in the primary screens. Meanwhile, the currently known effective antibiotics used to treat bartonellosis such as doxycycline, azithromycin, gentamicin, rifampin etc. were included as control drugs for comparison (Table 1). In addition, we included previously identified FDA-approved drugs that were effective against $B$. henselae such as daptomycin, methylene blue, miconazole and nitrofurantoin [22] as controls (Table 1). All these antibiotics were used at $20 \mu \mathrm{M}$. In the primary screens, 32 of the 149 essential oil collection were found to have good activity against stationary phase $B$. henselae both at the concentration of $0.5 \%$ and $0.25 \%$, and thus were selected as 
top hits. According to our previous experience, some compounds in the essential oils can cause interference with the SYBR Green I/PI assay because of color and autofluorescence. Thus, we selected these 32 top hits for further validation by microscopic counting to confirm the SYBR Green I/PI plate reader results. The currently used antibiotics for bartonellosis treatment and the identified FDA-approved drugs effective against $B$. henselae were also included as controls for comparison at 20 $\mu \mathrm{M}$. Doxycycline as a control drug showed mild activity against stationary phase B. henselae (residual viability above 26\%) (Table 1). Antibiotics reported to have clinical improvement for Bartonella infection including moxifloxacin, gentamicin, azithromycin, and rifampin [23,24] showed relatively better activity (residual viability between $9 \%$ and $25 \%$ ) against stationary phase B. henselae than doxycycline. And FDA-approved drugs that we identified effective against stationary phase $B$. henselae (daptomycin, methylene blue, miconazole and nitrofurantoin) had better activity (residual viability between $8 \%$ and $19 \%$ ) than most of the five antibiotics mentioned above.

Table 1. Activity of top 32 active hits that had good activity against stationary phase B. henselae ${ }^{1}$.

\begin{tabular}{|c|c|c|c|c|c|}
\hline \multirow[t]{2}{*}{$\begin{array}{l}\text { Essential oils } \\
\text { and control } \\
\text { drugs }\end{array}$} & \multirow[t]{2}{*}{$\begin{array}{l}\text { Plant or } \\
\text { ingredients of } \\
\text { essential oils }\end{array}$} & \multicolumn{2}{|c|}{$\begin{array}{c}\text { Residual viability }(\%) \text { after } 0.5 \% \\
\text { EO or } 20 \mu \mathrm{M} \text { antibiotics } \\
\text { treatment }\end{array}$} & \multicolumn{2}{|c|}{$\begin{array}{l}\text { Residual viability (\%) after } \\
\quad 0.25 \% \text { EO treatment }\end{array}$} \\
\hline & & Plate reader $^{2}$ & Microscope $^{3}$ & Plate reader $^{2}$ & Microscope $^{3}$ \\
\hline $\begin{array}{l}\text { Drug free } \\
\text { control }\end{array}$ & & $74 \%$ & $74 \%$ & & \\
\hline Doxycycline & & $26 \%$ & $57 \%$ & & \\
\hline Gentamicin & & $9 \%$ & $35 \%$ & & \\
\hline Moxifloxacin & & $22 \%$ & $40 \%$ & & \\
\hline Azithromycin & & $23 \%$ & $67 \%$ & & \\
\hline Rifampin & & $25 \%$ & $44 \%$ & & \\
\hline Daptomycin & & $8 \%$ & $18 \%$ & & \\
\hline $\begin{array}{c}\text { Methylene } \\
\text { Blue }\end{array}$ & & $16 \%$ & $27 \%$ & & \\
\hline Nitrofurantoin & & $18 \%$ & $50 \%$ & & \\
\hline Miconazole & & $19 \%$ & $44 \%$ & & \\
\hline Frankincense & Boswellia serrata & $5 \%$ & $11 \%$ & $6 \%$ & $10 \%$ \\
\hline Ylang ylang & Cananga odorata & $5 \%$ & $9 \%$ & $8 \%$ & $10 \%$ \\
\hline
\end{tabular}




\begin{tabular}{|c|c|c|c|c|c|}
\hline Tangerine & Citrus reticulata & $6 \%$ & $6 \%$ & $5 \%$ & $12 \%$ \\
\hline Bergamot & Citrus bergamia & $6 \%$ & $18 \%$ & $10 \%$ & $15 \%$ \\
\hline $\begin{array}{c}\text { Marjoram } \\
\text { (sweet) }\end{array}$ & $\begin{array}{l}\text { Origanum } \\
\text { marjorana }\end{array}$ & $6 \%$ & $13 \%$ & $5 \%$ & $15 \%$ \\
\hline Cajeput & $\begin{array}{c}\text { Melaleuca } \\
\text { cajeputi }\end{array}$ & $7 \%$ & $21 \%$ & $9 \%$ & $21 \%$ \\
\hline Lemon & Citrus limonum & $7 \%$ & $10 \%$ & $4 \%$ & $11 \%$ \\
\hline Oregano & $\begin{array}{c}\text { Origanum } \\
\text { vulgare hirtum }\end{array}$ & $7 \%$ & $7 \%$ & $7 \%$ & $20 \%$ \\
\hline $\begin{array}{l}\text { Geranium } \\
\text { bourbon }\end{array}$ & $\begin{array}{c}\text { Pelargonium } \\
\text { graveolens }\end{array}$ & $8 \%$ & $20 \%$ & $11 \%$ & $22 \%$ \\
\hline Tea tree & $\begin{array}{l}\text { Melaleuca } \\
\text { alternifolia }\end{array}$ & $8 \%$ & $12 \%$ & $5 \%$ & $25 \%$ \\
\hline Fir needle & Abies siberica & $8 \%$ & $25 \%$ & $10 \%$ & $26 \%$ \\
\hline Stress relief & $\begin{array}{c}\text { synergy blend } \\
\text { of essential oils } \\
\text { of bergamot, } \\
\text { patchouli, } \\
\text { sweet orange, } \\
\text { ylang ylang, } \\
\text { pink } \\
\text { grapefruit, } \\
\text { gurjum }\end{array}$ & $8 \%$ & $15 \%$ & $6 \%$ & $12 \%$ \\
\hline $\begin{array}{c}\text { Mountain } \\
\text { savory (winter) }\end{array}$ & $\begin{array}{l}\text { Satureja } \\
\text { montana }\end{array}$ & $8 \%$ & $25 \%$ & $21 \%$ & $32 \%$ \\
\hline Bandit & $\begin{array}{l}\text { synergy blend } \\
\text { of essential oils } \\
\text { of clove, } \\
\text { cinnamon, } \\
\text { lemon, } \\
\text { rosemary, } \\
\text { eucalyptus }\end{array}$ & $8 \%$ & $8 \%$ & $12 \%$ & $20 \%$ \\
\hline Cinnamon leaf & $\begin{array}{l}\text { Cinnamomum } \\
\text { zeylanicum }\end{array}$ & $8 \%$ & $35 \%$ & $10 \%$ & $25 \%$ \\
\hline Citronella & $\begin{array}{l}\text { Cymbopogon } \\
\text { winterianus }\end{array}$ & $8 \%$ & $15 \%$ & $12 \%$ & $23 \%$ \\
\hline Health shield & $\begin{array}{c}\text { blend of } \\
\text { cinnamon, } \\
\text { clove, }\end{array}$ & $9 \%$ & $18 \%$ & $17 \%$ & $20 \%$ \\
\hline
\end{tabular}




\begin{tabular}{|c|c|c|c|c|c|}
\hline & $\begin{array}{l}\text { eucalyptus, } \\
\text { lemon and } \\
\text { rosemary oils }\end{array}$ & & & & \\
\hline Spearmint & Mentha spicata & $9 \%$ & $9 \%$ & $4 \%$ & $20 \%$ \\
\hline Ho wood & $\begin{array}{c}\text { Cinnamomum } \\
\text { camphora }\end{array}$ & $9 \%$ & $20 \%$ & $11 \%$ & $29 \%$ \\
\hline $\begin{array}{c}\text { Tic Tox aux } \\
\text { huiles } \\
\text { essentielles }\end{array}$ & $\begin{array}{c}\text { blend of } \\
\text { essential oils of } \\
\text { savory, sage } \\
\text { officinale, wild } \\
\text { chamomile, } \\
\text { clove, compact } \\
\text { oregano, } \\
\text { cinnamon and } \\
\text { niaouli }\end{array}$ & $11 \%$ & $21 \%$ & $14 \%$ & $14 \%$ \\
\hline Citrus blast & $\begin{array}{l}\text { synergy blend } \\
\text { of Citrus sinesis, } \\
\text { Citrus limonum, } \\
\text { Citrus reticulata } \\
\text { blanco var } \\
\text { tangerina, } \\
\text { Citrus bergamia, } \\
\text { Citrus } \\
\text { reticulata, } \\
\text { Citrus } \\
\text { clementina, } \\
\text { Vanilla } \\
\text { planifolia }\end{array}$ & $11 \%$ & $13 \%$ & $11 \%$ & $30 \%$ \\
\hline Elemi & $\begin{array}{l}\text { Canarium } \\
\text { iuzonicum }\end{array}$ & $12 \%$ & $25 \%$ & $14 \%$ & $32 \%$ \\
\hline Vetiver & $\begin{array}{l}\text { Vetiveria } \\
\text { zizanoides }\end{array}$ & $12 \%$ & $26 \%$ & $8 \%$ & $18 \%$ \\
\hline Grapefruit & Citrus paradisi & $12 \%$ & $35 \%$ & $11 \%$ & $36 \%$ \\
\hline Clove bud 1 & $\begin{array}{c}\text { Eugenia } \\
\text { caryophyllata }\end{array}$ & $13 \%$ & $36 \%$ & $9 \%$ & $23 \%$ \\
\hline Deep forest & $\begin{array}{c}\text { synergy blend } \\
\text { of Abies sibirica } \\
\text { ledeb, Abies alba, } \\
\text { Pinus sylvestris, } \\
\text { Cupressus } \\
\text { sempervirens, }\end{array}$ & $13 \%$ & $20 \%$ & $12 \%$ & $50 \%$ \\
\hline
\end{tabular}




\begin{tabular}{|c|c|c|c|c|c|}
\hline & Cedrus deodora & & & & \\
\hline Geranium & $\begin{array}{c}\text { Pelargonlum } \\
\text { asperum }\end{array}$ & $14 \%$ & $23 \%$ & $15 \%$ & $20 \%$ \\
\hline Clove bud 2 & $\begin{array}{c}\text { Syzygium } \\
\text { aromaticum L }\end{array}$ & $15 \%$ & $15 \%$ & $14 \%$ & $18 \%$ \\
\hline Oil of oregano & $\begin{array}{c}\text { Origanum } \\
\text { vulgare hirtum }\end{array}$ & $15 \%$ & $52 \%$ & $19 \%$ & $55 \%$ \\
\hline Allspice & $\begin{array}{l}\text { Pimenta } \\
\text { officicalis }\end{array}$ & $16 \%$ & $35 \%$ & $6 \%$ & $30 \%$ \\
\hline Cedarwood & Cedrus deodora & $17 \%$ & $53 \%$ & $10 \%$ & $23 \%$ \\
\hline $\begin{array}{l}\text { Cinnamon } \\
\text { bark }\end{array}$ & $\begin{array}{c}\text { Cinnamomum } \\
\text { zeylanicum }\end{array}$ & $18 \%$ & $40 \%$ & $13 \%$ & $45 \%$ \\
\hline
\end{tabular}

${ }^{1}$ A 5-day-old stationary phase B. henselae culture was treated with essential oils or control drugs for 3 days. Control drugs used for comparison were shown in bold.

${ }^{2}$ Residual viability was calculated according to the regression equation and the ratio of Green/Red fluorescence obtained by SYBR Green I/PI assay.

${ }^{3}$ Residual viability was assayed by fluorescence microscope counting.

Among the 32 top hits that had better activity (residual viability between $5 \%$ and $21 \%$ ) against stationary phase $B$. henselae than most control antibiotics, the most active essential oils were ylang ylang, lemon, stress relief, health shield, Tic Tox aux huiles essentielles, geranium essential oil, clove bud, and cedarwood because of their remarkable activity at $0.25 \%$, as shown by red (dead) cells in fluorescence microscope observation (Figure 1). Essential oils made from oregano ("oregano" and "oil of oregano") and cinnamon ( "cinnamon leaf" and "cinnamon bark") were all shown to be active against stationary phase $B$. henselae, which have already been identified effective against stationary phase B. burgdorferi in our previous study [19]. Some essential oils extracted from plants of the same genus as oregano or cinnamon also exhibited good activity against stationary phase $B$. henselae. For example, ho wood, which was also extracted from Cinnamomum spp. as cinnamon essential oils was shown to be active. And marjoram (sweet), which was extracted from Origanum spp. as oregano essential oils was also active. In addition, essential oils extracted from Citrus spp. including tangerine, bergamot, lemon and grapefruit all exhibited strong activity against stationary phase $B$. henselae, and the same for essential oils extracted from Pelargonium spp. (geranium bourbon and geranium essential oil) and Melaleuca spp. (cajeput and tea tree). Many synergy blend essential oils including "stress relief", "bandit", "health shield", "Tic Tox", "citrus blast" and "deep forest" exhibited strong activity against stationary phase $B$. henselae because their effective ingredients were shown to be active by other single essential oils, such as clove, ylang ylang, lemon, bergamot, grapefruit, cinnamon, oregano and fir needle. Control drugs including doxycycline (DOX) and azithromycin (AZI) exhibited poor activity against stationary phase $B$. henselae as shown by green (live) cells in fluorescence microscope observation, other antibiotics including gentamicin (GEN), moxifloxacin (MXF), rifampin (RIF), daptomycin (DAP), methylene blue, nitrofurantoin (NIT) and miconazole showed better activity, while not as good as most of the 32 top hits (Figure 1). 
Drug free
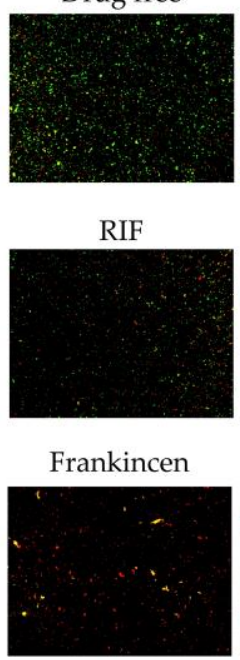

Cajeput

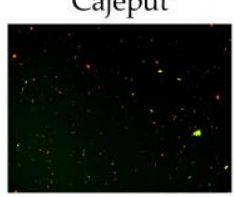

Fir needle

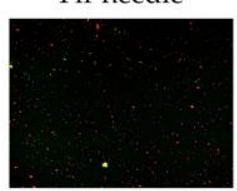

Citronella

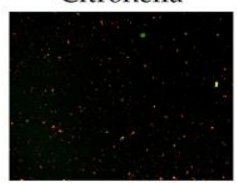

Citrus blast

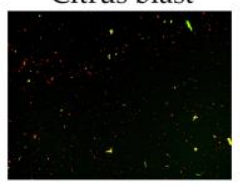

Deep forest

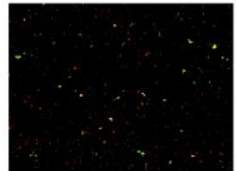

Cinnamon bark

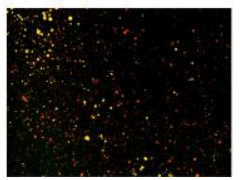

DOX

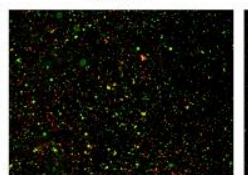

DAP

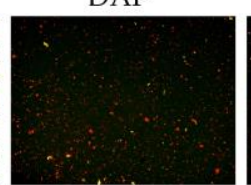

Ylang ylang

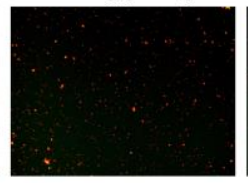

Lemon

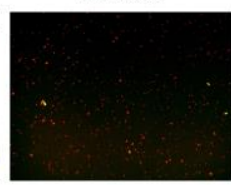

Stress relief

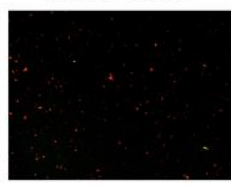

Health shield

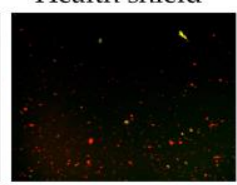

Elemi

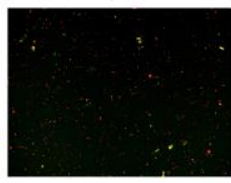

Geranium

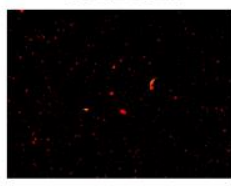

Cedarwood

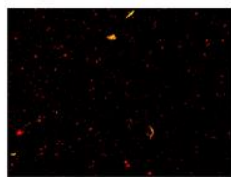

GEN

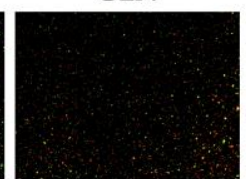

Methylene blue

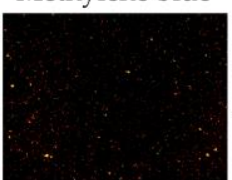

Tangerine

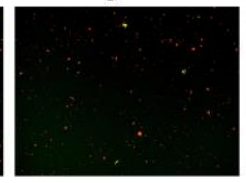

Oregano

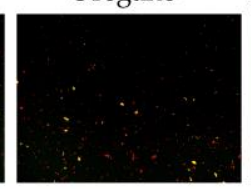

Mountain savory (winter)

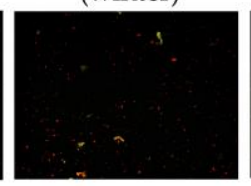

Spearmint

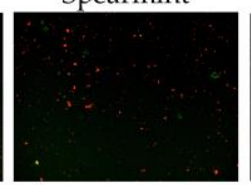

Vetiver

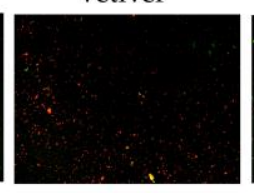

Clove bud 2

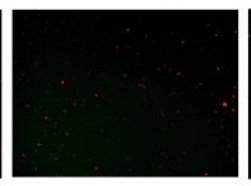

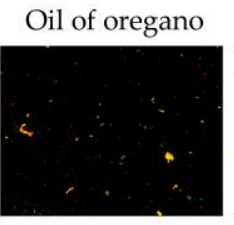

AZI
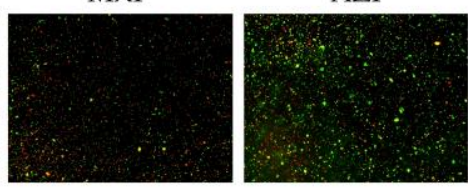

Miconazole
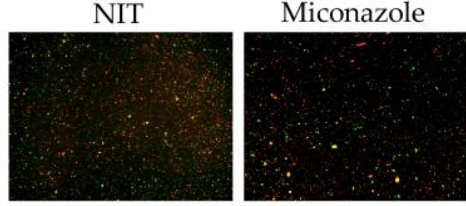

Bergamo

Marjoram (sweet)
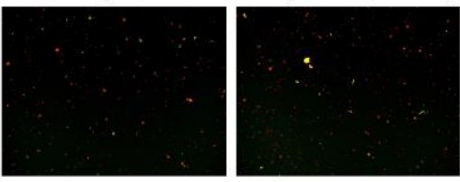

Geranium bourbon
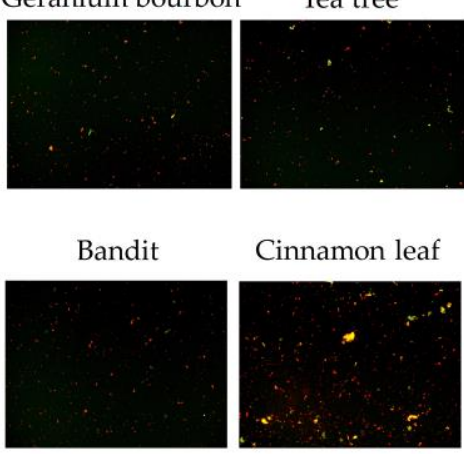

Cinnamon leaf

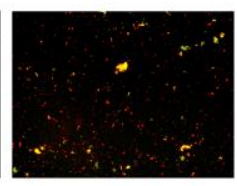

Ho wood Tic Tox aux huiles
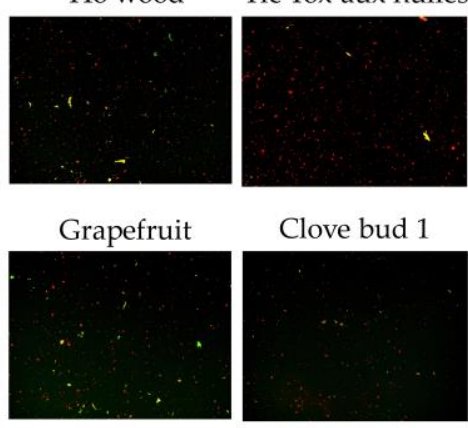

Allspice

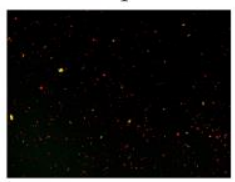

Figure 1. Effect of 32 top hits of essential oils against stationary phase B. henselae JK53 in comparison with control drugs. A 5-day-old stationary phase B. henselae JK53 culture was treated with $0.25 \%$ (v/v) essential oils or control drugs $(20 \mu \mathrm{M})$ for 3 days followed by SYBR Green I/PI viability assay and fluorescence microscopy. 


\subsection{MIC determination of active hits}

The essential oils listed above were active against the non-growing stationary phase $B$. henselae (Table 1 and Figure 1), and it was necessary to determine the MICs of these active drugs against log phase growing $B$. henselae. The MICs of essential oils for B. henselae were determined by the standard microdilution method, as described in our previous study [25]. As shown in Table 2, cinnamon bark was the most active essential oil among these 32 hits, capable of inhibiting visible B. henselae proliferation at the lowest concentration of essential oils tested $(0.008 \%)$. The health shield, a blend of many active compounds against non-growing $B$. henselae were also highly active against growing $B$. henselae, which could inhibit B. henselae proliferation at a concentration of $0.008 \%-0.016 \%$. And the growth of $B$. henselae was efficiently suppressed by bandit, elemi, mountain savory (winter), cedarwood and two oregano essential oils at $0.016 \%-0.032 \%$, and by ylang ylang, citronella, clove bud, geranium bourbon, allspice, vetiver, cinnamon leaf and geranium essential oil at 0.032\%-0.063\%. Other single essential oils including bergamot, cajeput, marjoram (sweet), fir needle, grapefruit as well as blend essential oils including stress relief, citrus blast and deep forest were also active with MIC values of $0.063 \%-0.125 \%$. B. henselae growing cells were also susceptible to spearmint, tangerine, tea tree, lemon, ho wood, frankincense and Tic Tox aux huiles essentielles at a concentration of $0.125 \%-0.25 \%$, though relatively higher than others.

Table 2. Minimal inhibitory concentrations (MICs) of top hit essential oils against B. henselae ${ }^{1}$.

\begin{tabular}{|c|c|c|}
\hline Essential oils & $\begin{array}{c}\text { Plant or ingredients of } \\
\text { essential oils }\end{array}$ & $\operatorname{MIC~(v/v)~}$ \\
\hline Cinnamon bark & Cinnamomum zeylanicum & $<0.008 \%$ \\
\hline Health shield & $\begin{array}{l}\text { blend of cinnamon, clove, } \\
\text { eucalyptus, lemon and } \\
\text { rosemary oils }\end{array}$ & $0.008 \%-0.016 \%$ \\
\hline Bandit & $\begin{array}{l}\text { synergy blend of essential } \\
\text { oils of clove, cinnamon, } \\
\text { lemon, rosemary, eucalyptus }\end{array}$ & $0.016 \%-0.032 \%$ \\
\hline Oregano & Origanum vulgare hirtum & $0.016 \%-0.032 \%$ \\
\hline Elemi & Canarium iuzonicum & $0.016 \%-0.032 \%$ \\
\hline Oil of oregano & Origanum vulgare hirtum & $0.016 \%-0.032 \%$ \\
\hline Mountain savory (winter) & Satureja montana & $0.016 \%-0.032 \%$ \\
\hline Cedarwood & Cedrus deodora & $0.016 \%-0.032 \%$ \\
\hline Ylang ylang & Cananga odorata & $0.032 \%-0.063 \%$ \\
\hline Citronella & Cymbopogon winterianus & $0.032 \%-0.063 \%$ \\
\hline Clove bud 1 & Eugenia caryophyllata & $0.032 \%-0.063 \%$ \\
\hline Clove bud 2 & Syzygium aromaticum $L$ & $0.032 \%-0.063 \%$ \\
\hline Geranium bourbon & Pelargonium graveolens & $0.032 \%-0.063 \%$ \\
\hline Allspice & Pimenta officicalis & $0.032 \%-0.063 \%$ \\
\hline Vetiver & Vetiveria zizanoides & $0.032 \%-0.063 \%$ \\
\hline Cinnamon leaf & Cinnamomum zeylanicum & $0.032 \%-0.063 \%$ \\
\hline
\end{tabular}




\begin{tabular}{|c|c|c|}
\hline Geranium & Pelargonlum asperum & $0.032 \%-0.063 \%$ \\
\hline Stress relief & $\begin{array}{l}\text { synergy blend of essential } \\
\text { oils of bergamot, patchouli, } \\
\text { sweet orange, ylang ylang, } \\
\text { pink grapefruit, gurjum }\end{array}$ & $0.063 \%-0.125 \%$ \\
\hline Bergamot & Citrus bergamia & $0.063 \%-0.125 \%$ \\
\hline Cajeput & Melaleuca cajeputi & $0.063 \%-0.125 \%$ \\
\hline Marjoram (sweet) & Origanum marjorana & $0.063 \%-0.125 \%$ \\
\hline Citrus blast & $\begin{array}{l}\text { synergy blend of essential } \\
\text { oils of bergamot, patchouli, } \\
\text { sweet orange, ylang ylang, } \\
\text { pink grapefruit, gurjum }\end{array}$ & $0.063 \%-0.125 \%$ \\
\hline Deep forest & $\begin{array}{c}\text { synergy blend of Abies } \\
\text { sibirica ledeb, Abies alba, Pinus } \\
\text { sylvestris, Cupressus } \\
\text { sempervirens, Cedrus deodora }\end{array}$ & $0.063 \%-0.125 \%$ \\
\hline Fir needle & Abies siberica & $0.063 \%-0.125 \%$ \\
\hline Grapefruit & Citrus paradisi & $0.063 \%-0.125 \%$ \\
\hline Spearmint & Mentha spicata & $0.125 \%-0.25 \%$ \\
\hline Tangerine & Citrus reticulata & $0.125 \%-0.25 \%$ \\
\hline Tea tree & Melaleuca alternifolia & $0.125 \%-0.25 \%$ \\
\hline Lemon & Citrus limonum & $0.125 \%-0.25 \%$ \\
\hline Ho wood & Cinnamomum camphora & $0.125 \%-0.25 \%$ \\
\hline Frankincense & Boswellia serrata & $0.125 \%-0.25 \%$ \\
\hline $\begin{array}{l}\text { Tic Tox aux huiles } \\
\text { essentielles }\end{array}$ & $\begin{array}{c}\text { blend of essential oils of } \\
\text { savory, sage officinale, wild } \\
\text { chamomile, clove, compact } \\
\text { oregano, cinnamon and } \\
\text { niaouli }\end{array}$ & $0.125 \%-0.25 \%$ \\
\hline
\end{tabular}

${ }^{1}$ The MIC testing for B. henselae was set up as described in Methods.

\subsection{Time-kill curves of active hits}

Having obtained 32 top hits by primary screens, we performed a time-kill drug exposure assay against a 5-day-old stationary phase $B$. henselae culture at a lower concentration of these active essential oils. Here we just selected single essential oil samples for drug exposure assay in order to better evaluate and compare the activity of antimicrobial components among different essential oils. All selected 25 essential oils were applied at $0.032 \%(\mathrm{v} / \mathrm{v})$, respectively. Clinically used antibiotics and the previously identified effective FDA-approved drugs against $B$. henselae were used at their Cmax as controls. As shown in Table 3 and Figure $2 b$ \& 2c, oregano, cinnamon bark and mountain savory (winter) were the most active essential oils that rapidly killed $B$. henselae with no detectable CFU after 1-day exposure. Other active hits, including clove bud 2, allspice, geranium, and cinnamon leaf could eradicate $B$. henselae cells without viable cells being recovered after 3-day drug 
exposure. Geranium bourbon and clove bud 1 also showed excellent activity which could kill all bacteria by Day 5 , followed by elemi, vetiver, citronella and ylang ylang that eradicated all $B$. henselae cells by Day 7.

As shown in Table 3, grapefruit, tangerine, bergamot, fir needle, frankincense and ho wood were also quite active, reducing $5 \log 10 \mathrm{CFU} / \mathrm{mL}$ after 7-day exposure. And lemon, as well as marjoram (sweet) also had the capability of killing stationary phase B. henselae and reduced the bacterial count by approximately $3 \log 10 \mathrm{CFU} / \mathrm{mL}$ in 7 days. However, cajeput, tea tree, cedarwood and spearmint showed poor activity. Compared with drug free control, as shown in Figure 2a, some clinically used antibiotics for Bartonella treatment, such as azithromycin and doxycycline, had poor activity in killing $B$. henselae, achieving approximately $1 \log 10 \mathrm{CFU} / \mathrm{mL}$ decrease after 7 -day drug exposure. Gentamicin and rifampin showed better activity than azithromycin and doxycycline when used at their Cmax, which could eradicate all B. henselae cells respectively by Day 3 and Day 7. Other FDA-approved drugs effective against $B$. henselae including daptomycin and methylene blue had good activity that led to eradication of $B$. henselae cells after 1-day or 5-day exposure, respectively, while miconazole did not kill all B. henselae cells by Day 7 when used at Cmax.

a

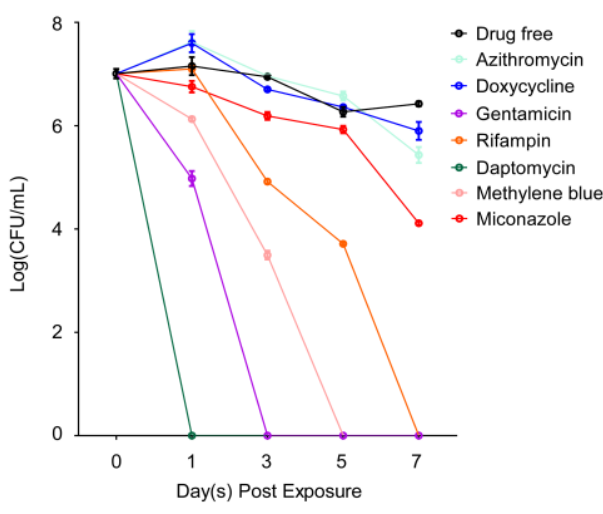

b

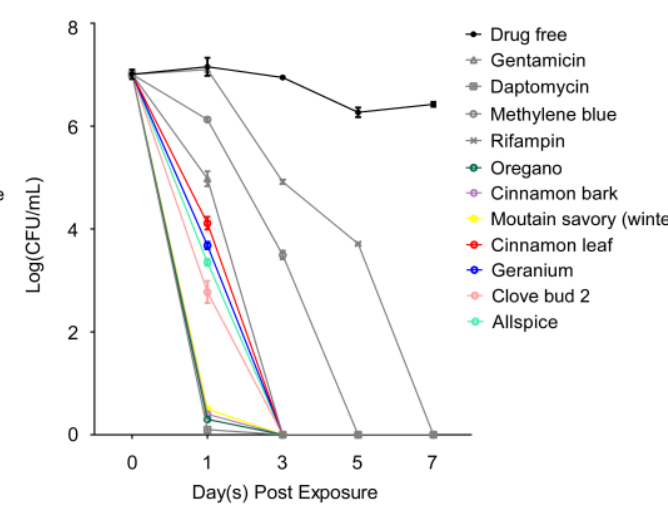

C

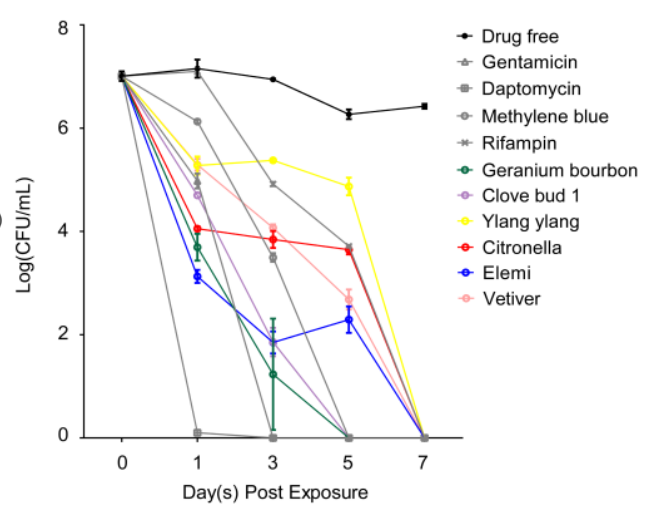

Figure 2. Time-kill curves for essential oils treatment of 5-day-old stationary phase B. henselae in comparison with control drugs. (a) Time-kill curves for control antibiotic treatment. (b-c) Time-kill curves for essential oils treatment. Drug free control, daptomycin, gentamicin, methylene blue and rifampin treatment were the same of $\mathrm{a}, \mathrm{b}$ and $\mathrm{c}$. The essential oils or control antibiotics were added to the stationary phase culture respectively at time point 0 , and at different times of drug exposure (Day 1, Day 3, Day 5 and Day 7), portions of bacteria were removed and washed and plated on Columbia blood agar for CFU counts. The essential oil concentration used in this experiment was $0.032 \%(\mathrm{v} / \mathrm{v})$. Drug concentrations used in this experiment were based on their Cmax and are as follows: $2.4 \mu \mathrm{g} / \mathrm{mL}$ doxycycline, $0.2 \mu \mathrm{g} / \mathrm{mL}$ azithromycin, $10 \mu \mathrm{g} / \mathrm{mL}$ gentamicin, $7.8 \mu \mathrm{g} / \mathrm{mL}$ rifampin, $60 \mu \mathrm{g} / \mathrm{mL}$ daptomycin, $2.9 \mu \mathrm{g} / \mathrm{mL}$ methylene blue, and $6.3 \mu \mathrm{g} / \mathrm{mL}$ miconazole.

Table 3. Drug exposure assay of selected active essential oils against a 5-day-old stationary phase $B$. henselae culture ${ }^{1}$. 


\begin{tabular}{|c|c|c|c|c|}
\hline $\begin{array}{l}\text { and control } \\
\text { drugs }^{2}\end{array}$ & 1 Day & 3 Day & 5 Day & 7 Day \\
\hline $\begin{array}{l}\text { Drug free } \\
\text { control }\end{array}$ & $1.50 \pm 0.53 \times 10^{7}$ & $8.83 \pm 0.29 \times 10^{6}$ & $1.88 \pm 0.40 \times 10^{6}$ & $2.67 \pm 0.29 \times 10^{6}$ \\
\hline Doxycycline & $4.17 \pm 1.44 \times 10^{7}$ & $5.07 \pm 0.38 \times 10^{6}$ & $2.30 \pm 0.10 \times 10^{6}$ & $8.33 \pm 2.89 \times 10^{5}$ \\
\hline Azithromycin & $4.50 \pm 2.00 \times 10^{7}$ & $9.17 \pm 0.29 \times 10^{6}$ & $3.80 \pm 0.72 \times 10^{6}$ & $2.83 \pm 1.04 \times 10^{5}$ \\
\hline Gentamicin & $9.83 \pm 2.93 \times 10^{4}$ & 0 & 0 & 0 \\
\hline Rifampin & $1.27 \pm 0.15 \times 10^{7}$ & $8.33 \pm 0.76 \times 10^{4}$ & $5.17 \pm 0.29 \times 10^{3}$ & 0 \\
\hline Daptomycin & 0 & 0 & 0 & 0 \\
\hline Methylene blue & $1.35 \pm 0.13 \times 10^{6}$ & $3.17 \pm 0.58 \times 10^{3}$ & 0 & 0 \\
\hline Miconazole & $5.83 \pm 1.53 \times 10^{6}$ & $1.57 \pm 0.28 \times 10^{6}$ & $8.50 \pm 1.32 \times 10^{5}$ & $1.30 \pm 0.10 \times 10^{4}$ \\
\hline Oregano & 0 & 0 & 0 & 0 \\
\hline Cinnamon bark & 0 & 0 & 0 & 0 \\
\hline $\begin{array}{c}\text { Mountain } \\
\text { savory (winter) }\end{array}$ & 0 & 0 & 0 & 0 \\
\hline Clove bud 2 & $6.50 \pm 3.46 \times 10^{2}$ & 0 & 0 & 0 \\
\hline Allspice & $2.27 \pm 0.33 \times 10^{3}$ & 0 & 0 & 0 \\
\hline Geranium & $4.83 \pm 0.76 \times 10^{3}$ & 0 & 0 & 0 \\
\hline Cinnamon leaf & $1.33 \pm 0.35 \times 10^{4}$ & 0 & 0 & 0 \\
\hline $\begin{array}{l}\text { Geranium } \\
\text { bourbon }\end{array}$ & $5.50 \pm 2.65 \times 10^{3}$ & $5.00 \pm 5.00 \times 10$ & 0 & 0 \\
\hline Clove bud 1 & $5.00 \pm 0.00 \times 10^{4}$ & $8.33 \pm 5.77 \times 10$ & 0 & 0 \\
\hline Elemi & $1.38 \pm 0.42 \times 10^{3}$ & $5.00 \pm 5.00 \times 10$ & $2.17 \pm 1.04 \times 10^{2}$ & 0 \\
\hline Vetiver & $2.00 \pm 0.50 \times 10^{5}$ & $1.18 \pm 0.19 \times 10^{4}$ & $5.17 \pm 2.47 \times 10^{2}$ & 0 \\
\hline Citronella & $1.13 \pm 0.12 \times 10^{4}$ & $7.33 \pm 2.84 \times 10^{3}$ & $4.50 \pm 0.87 \times 10^{3}$ & 0 \\
\hline Ylang ylang & $2.00 \pm 0.87 \times 10^{5}$ & $2.38 \pm 0.19 \times 10^{5}$ & $7.83 \pm 3.01 \times 10^{4}$ & 0 \\
\hline Grapefruit & $1.02 \pm 0.19 \times 10^{4}$ & $3.17 \pm 1.89 \times 10^{4}$ & $5.33 \pm 1.26 \times 10^{3}$ & $6.67 \pm 5.77 \times 10$ \\
\hline Tangerine & $3.17 \pm 0.29 \times 10^{4}$ & $2.08 \pm 0.58 \times 10^{4}$ & $4.50 \pm 2.29 \times 10^{3}$ & $6.67 \pm 5.77 \times 10$ \\
\hline
\end{tabular}




\begin{tabular}{ccccc}
\hline Bergamot & $8.17 \pm 2.25 \times 10^{3}$ & $2.62 \pm 0.35 \times 10^{4}$ & $6.83 \pm 0.76 \times 10^{3}$ & $1.67 \pm 0.58 \times 10^{2}$ \\
\hline Fir needle & $4.17 \pm 1.61 \times 10^{3}$ & $2.32 \pm 0.41 \times 10^{4}$ & $1.10 \pm 0.13 \times 10^{4}$ & $1.67 \pm 0.58 \times 10^{2}$ \\
\hline Frankincense & $1.35 \pm 0.22 \times 10^{5}$ & $8.17 \pm 1.53 \times 10^{5}$ & $1.48 \pm 0.29 \times 10^{6}$ & $1.83 \pm 0.76 \times 10^{2}$ \\
\hline Ho wood & $5.00 \pm 0.50 \times 10^{6}$ & $7.50 \pm 2.65 \times 10^{5}$ & $1.37 \pm 0.28 \times 10^{5}$ & $4.17 \pm 1.44 \times 10^{2}$ \\
\hline Lemon & $3.17 \pm 1.15 \times 10^{4}$ & $1.03 \pm 0.28 \times 10^{5}$ & $8.67 \pm 0.76 \times 10^{4}$ & $4.33 \pm 2.31 \times 10^{3}$ \\
\hline Marjoram & $2.17 \pm 1.53 \times 10^{5}$ & $2.13 \pm 0.28 \times 10^{6}$ & $2.22 \pm 0.25 \times 10^{6}$ & $7.50 \pm 1.32 \times 10^{3}$ \\
(Sweet) & $2.50 \pm 0.87 \times 10^{6}$ & $9.43 \pm 0.40 \times 10^{6}$ & $3.20 \pm 0.26 \times 10^{6}$ & $1.62 \pm 0.25 \times 10^{5}$ \\
\hline Cajeput & $8.00 \pm 2.18 \times 10^{5}$ & $9.33 \pm 0.29 \times 10^{6}$ & $3.97 \pm 0.45 \times 10^{6}$ & $3.17 \pm 0.76 \times 10^{6}$ \\
\hline Tea tree & $2.33 \pm 2.31 \times 10^{5}$ & $2.73 \pm 0.33 \times 10^{6}$ & $3.40 \pm 0.36 \times 10^{6}$ & $3.52 \pm 0.18 \times 10^{6}$ \\
\hline Cedarwood & $4.33 \pm 1.26 \times 10^{5}$ & $9.17 \pm 0.29 \times 10^{6}$ & $3.67 \pm 0.58 \times 10^{6}$ & $3.68 \pm 0.38 \times 10^{6}$ \\
\hline Spearmint & 2.53 & & \\
\hline
\end{tabular}

${ }^{1}$ A 5-day-old stationary phase $B$. henselae culture was treated with essential oils or control drugs. Control drugs used for comparison were shown in bold. The beginning CFU for the 5-day-old stationary phase B. henselae culture was about $1 \times 10^{7} \mathrm{CFU} / \mathrm{mL}$. At different times of drug exposure (Day 1, Day 3, Day 5 and Day 7),

portions of bacteria were removed and washed and plated on Columbia blood agar for CFU counts.

2 The essential oil concentration used in this experiment was $0.032 \%(\mathrm{v} / \mathrm{v})$. Drug concentrations used in this experiment were based on their Cmax and are as follows: $2.4 \mu \mathrm{g} / \mathrm{mL}$ doxycycline, $0.2 \mu \mathrm{g} / \mathrm{mL}$ azithromycin, 10 $\mu \mathrm{g} / \mathrm{mL}$ gentamicin, $7.8 \mu \mathrm{g} / \mathrm{mL}$ rifampin, $60 \mu \mathrm{g} / \mathrm{mL}$ daptomycin, $2.9 \mu \mathrm{g} / \mathrm{mL}$ methylene blue, and $6.3 \mu \mathrm{g} / \mathrm{mL}$ miconazole.

2.4. Carvacrol and cinnamaldehyde as highly potent active ingredient of essential oils against stationary phase B. henselae

Our previous studies have identified two components, carvacrol and cinnamaldehyde, as highly potent active ingredients of oregano and cinnamon bark essential oils, respectively, which were effective against B. burgdorferi $[19,20]$. As shown above, oregano and cinnamon bark essential oils were also highly active to kill B. henselae, so we tested carvacrol and cinnamaldehyde, two major constituents of these two active essential oils, for their antimicrobial activity against B. henselae. Carvacrol and cinnamaldehyde were applied at two concentrations, $0.01 \%(\mathrm{v} / \mathrm{v})$ and $0.005 \%(\mathrm{v} / \mathrm{v})$, respectively, for the drug exposure assay against a 5-day-old stationary phase B. henselae culture. Clinically used antibiotics and the previously identified effective FDA-approved drugs against $B$. henselae were used at their Cmax as controls. As shown in Figure 3, 0.01\% carvacrol could eradicate $B$. henselae cells without viable cells being recovered after 5-day drug exposure. And 0.005\% carvacrol led to $2 \log 10 \mathrm{CFU} / \mathrm{mL}$ reduction after 7-day exposure. Cinnamaldehyde was especially active such that it rapidly killed all stationary phase B. henselae cells with no detectable CFU after 1-day exposure when used at the concentration of $0.01 \%$. And $0.005 \%$ cinnamaldehyde could also eradicate all $B$. henselae cells after 3-day exposure. According to the concentration of original stock, 0.005\% carvacrol or cinnamaldehyde was approximately equal to $50 \mu \mathrm{g} / \mathrm{mL}$. So the antimicrobial activity of carvacrol and cinnamaldehyde against $B$. henselae was comparable to that of effective FDA-approved 
antibiotics against stationary phase $B$. henselae, including gentamicin, rifampin, daptomycin and methylene blue, which could eradicate all B. henselae cells within 7-day drug exposure when used at their Cmax.

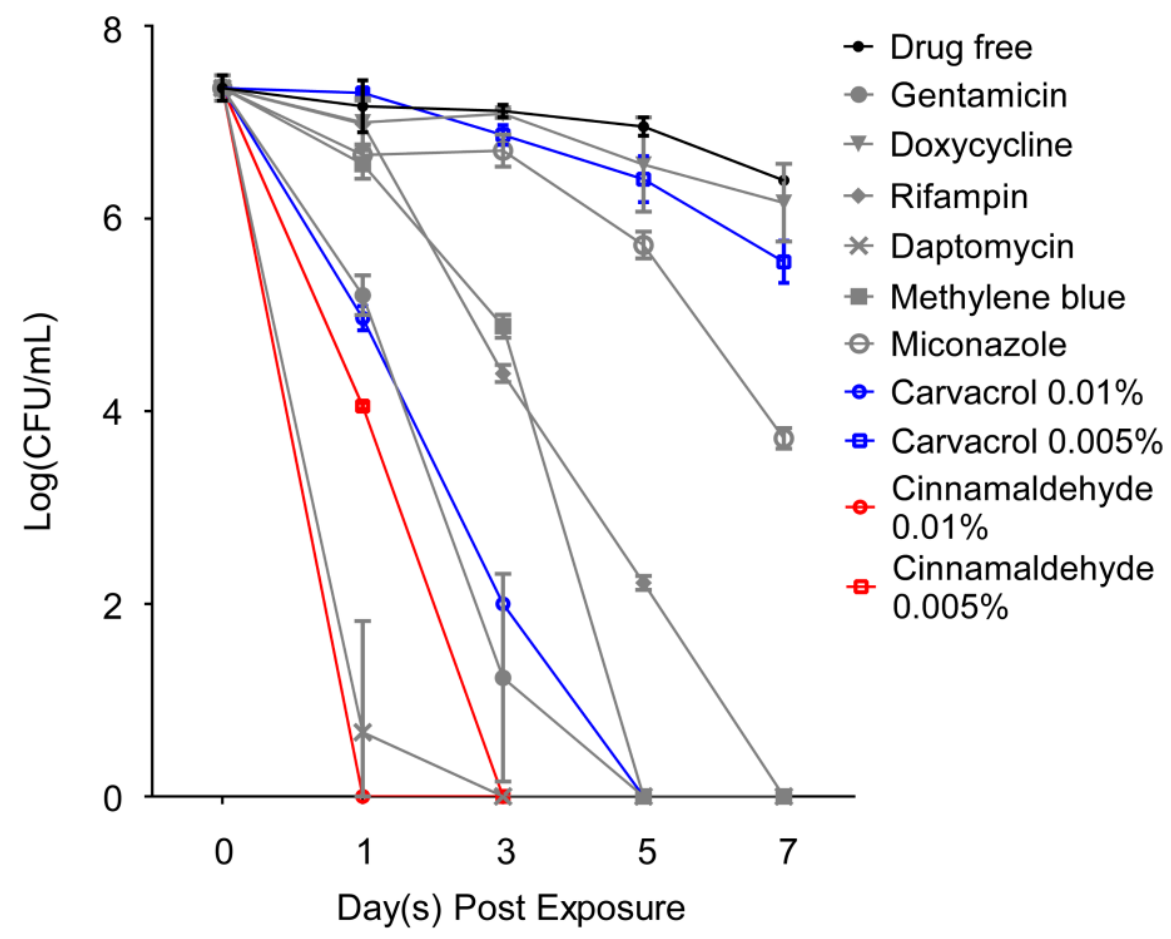

Figure 3. Time-kill curves for carvacrol and cinnamaldehyde treatment of 5-day-old stationary phase $B$. henselae in comparison with control drugs. Carvacrol, cinnamaldehyde or control antibiotics were added to the stationary phase culture respectively at time point 0 , and at different times of drug exposure (Day 1, Day 3, Day 5 and Day 7), portions of bacteria were removed and washed and plated on Columbia blood agar for CFU counts. The concentrations of carvacrol and cinnamaldehyde used were $0.01 \%(\mathrm{v} / \mathrm{v})$ and $0.005 \%(\mathrm{v} / \mathrm{v})$. Drug concentrations used in this experiment were based on their Cmax and are as follows: $2.4 \mu \mathrm{g} / \mathrm{mL}$ doxycycline, $10 \mu \mathrm{g} / \mathrm{mL}$ gentamicin, $7.8 \mu \mathrm{g} / \mathrm{mL}$ rifampin, $60 \mu \mathrm{g} / \mathrm{mL}$ daptomycin, $2.9 \mu \mathrm{g} / \mathrm{mL}$ methylene blue, and $6.3 \mu \mathrm{g} / \mathrm{mL}$ miconazole.

\section{Discussion}

In this study, we successfully applied the SYBR Green I/PI viability assay for high-throughput screen of essential oil collection for activity against stationary phase $B$. henselae as a model of persister drug screens. We identified 32 essential oils at $0.25 \%$ concentration which have better activity than the current clinically used antibiotics for bartonellosis treatment. These include four essential oils extracted from plants of genus Citrus (tangerine, bergamot, lemon and grapefruit), three from Origanum (two oregano essential oils and marjoram), three from Cinnamomum (cinnamon bark, cinnamon leaf and ho wood), two from Pelargonium (geranium bourbon and geranium essential oil) and two from Melaleuca (cajeput and tea tree). Among these 32 top hits, thirteen single essential oils could effectively kill all stationary B. henselae cells without CFU detected within 7-day drug exposure even at a low concentration of $0.032 \%(\mathrm{v} / \mathrm{v})$, where the essential oils of oregano, cinnamon bark and mountain savory (winter) were the most active ones that eradicated bacteria 
after 1-day exposure. Some essential oils that showed activity by primary screens exhibited poor activity in drug exposure assay, partly due to the volatility of essential oils during such a long incubation period. Carvacrol and cinnamaldehyde, two active ingredients of effective essential oils, oregano and cinnamon bark, respectively, were shown to be extremely active against stationary phase $B$. henselae that they could eradicate all bacterial cells within 7-day drug exposure even at a very low concentration $\leq 0.01 \%(\mathrm{v} / \mathrm{v})$. And the MIC determination showed the 32 active hits were not only active against stationary phase $B$. henselae but also effective in inhibiting the growth of log phase B. henselae, especially the essential oil of cinnamon bark.

The pattern that some different plant species of the same genus all possess the antimicrobial ability could serve as a guideline in our future study to obtain more active hits and decode the antimicrobial mechanism. Here, we identified Citrus, Cinnamomum, Origanum, Pelargonium and Melaleuca as potential genera that might include more plants active against $B$. henselae. Citrus plants constitute one of the most valuable and important sources of essential oil served in food processing and medical use. Citrus limonum essential oil was reported to have antimicrobial activities with preservative effect against Listeria monocytogenes inoculated in minced beef meat [26], and remarkable miticidal activity in vitro and in vivo applications against sarcoptic mange in rabbits [27]. Besides, the essential oil from Citrus limetta Risso peels could alleviate skin inflammation, both tested in vitro and in vivo [28], while the essential oil from Citrus aurantium L. var. amara Engl also had anti-inflammatory effect [29]. Citrus leaf extract was reported to reduce blood pressure and vascular damage in repeatedly heated palm oil diet-induced hypertensive rats [30]. These studies indicated Citrus plants could serve in different health care treatments including antimicrobial function. And our study was the first to identify their activity against $B$. henselae.

Previous studies have shown that oregano oil has antibacterial activity against three Gram-positive and two Gram-negative bacteria of their growing log phase [31]. It was also reported to be highly effective against stationary phase B. burgdorferi [19]. Cinnamon, clove bud and allspice were well-known as flavors for food processing, while they were both found to have excellent activity against B. burgdorferi stationary phase cells in vitro, even better than the persister drug daptomycin [19,20]. Allspice was also known to have antibacterial activities on many organisms [32]. Here, for the first time, we identified essential oils of oregano, cinnamon, clove bud and allspice as having highly potent activities against both $\log$ phase and stationary phase $B$. henselae. It is interesting to note that the high activity of these common essential oils against both Borrelia and Bartonella indicated that they had the potential to be active against both Borrelia and Bartonella persistent infections, which clinically may be present as coinfections [33] .

Other essential oils identified effective in our study have also been proved to have good biological activities by previous studies. It was reported that frankincense and geranium essential oils could suppress tumor progression through the regulation of the AMPK/mTOR pathway in breast cancer [34]. And geranium essential oil could eradicate enterococcal biofilm at concentration of $150 \mathrm{mg} / \mathrm{mL}$ without bacteria developing resistance to it, thus could be a possible alternative to other antimicrobials during endodontic procedures [35]. Frankincense was reported to have anti-inflammatory and antibacterial effects [36]. Mountain savory has been proved to be highly active against methicillin-resistant Staphylococcus aureus (MRSA), Salmonella typhimurium and L. monocytogenes [37-39]. According to previous studies, fir honeydew honey had strong antimicrobial activity against S. aureus, A. baumannii, P. aeruginosa, E. coli and kinds of fungi [40-42]. Ylang ylang 
products have a wide variety of bioactivities including antimicrobial, antibiofilm, anti-inflammatory, anti-vector, insect-repellent and so on, demonstrating it to be a useful plant to agriculture and medicine [43]. Citronella was reported to have antifungal and antibiofilm activity as well as antimicrobial activity against Staphylococcus [44,45]. Extracts from Canarium were proved to be active against MRSA and P. aeruginosa [46]. Essential oils of vetiver were active against S. aureus and showed good antifungal and cytotoxic activities [47,48]. And here, for the first time, we identified the remarkable activity of these essential oils active against both log phase and stationary phase $B$. henselae.

Although our study has identified many active hits from the essential oil collection, future studies are needed to identify the active ingredients of these active essential oils. Here we just tested two active ingredients, carvacrol and cinnamaldehyde, which showed excellent ability to eradicate stationary phase $B$. henselae even at a much lower concentration than correlated essential oil samples used. Some previous studies have identified main ingredients of some active essential oils such as mountain savory, thyme, lemongrass, limette and cumin, including geranial, $\beta$-pinene, thymol, $\gamma$-terpinene, citronellal and so on [49]. The antimicrobial activity of these components should be studied thoroughly in the future in order to determine their utility.

It is worth noticing that clinically used drugs for bartonellosis treatment including doxycycline, azithromycin, rifampin, moxifloxacin and gentamicin showed poor capability in eradicating stationary phase B. henselae cells (Table 1 and Figure 1), as they mainly target growing cells in log phase. The discrepant efficacies of antibiotics between in vitro MIC data and clinical data from patients with bartonellosis were also reported and the poor activities of current clinically used antibiotics against stationary phase $B$. henselae could partly explain for the treatment failure and persistence of infection [11]. The microscopic observation showed that stationary phase B. henselae cells tended to aggregate together (Figure 1), which might protect them from attack by antimicrobial agents. Bacterial cell membrane is a known target of some persister drugs, and it is interesting to note that essential oils are exactly agents targeting the membrane because of their lipophilicity. Due to this property, essential oils could exhibit much higher activity against stationary phase $B$. henselae in aggregated biofilm form than current clinically used antibiotics for Bartonella infections and could be considered promising candidates for further evaluation. But it should also be noted that the high lipophilicity of essential oils might cause B. henselae cells or biofilm structures to be dissolved, leading to reduction of the dead cell number and as a result, the residual viability percentage by the SYBR Green I/PI assay might be misinterpreted.

Another promising strategy for developing more effective treatment for bartonellosis is the drug combination of active essential oils with antibiotics to avoid resistance development and improve the efficacy of the treatment. Future studies are needed to evaluate drug combinations of two or more newly identified essential oil candidates with current clinically used drugs in combination, in order to better target diverse bacterial populations of different phases or forms that can happen in the host as indicated by the Yin-Yang model [13]. There were some previous studies of evaluating the antimicrobial activity of combined essential oil samples against multidrug resistance (MDR) E. coli, Klebsiella pneumoniae, MRSA, Staphylococcus epidermidis, Propionibacterium acnes, as well as airborne bacteria and fungi in hospital rooms, and some of the essential oil combinations did have better activity than used alone [50-52]. 
In this study, we identified a range of essential oils with high activity against stationary phase $B$. henselae in vitro. Because $B$. henselae can reside and propagate inside erythrocytes and/or endothelial cells in humans and animals [53,54], which could provide a shelter that protects them from the host immune responses and exposure to antibiotics, future studies are needed to evaluate the activities of selected essential oils against intracellular B. henselae. The active ingredients of some effective essential oils remain unknown, and neither are the pharmacokinetic profiles of these ingredients. These should be studied thoroughly in the future in order to help decode the antimicrobial mechanism and further evaluate their activity in vivo. Further validation using appropriate animal models of bartonellosis is required to assess the safety and efficacy of identified essential oils as well as to determine their effective dosage in vivo. Our study was performed with $B$. henselae and future studies are needed to test if the findings here apply to other closely related pathogenic Bartonella species, such as B. quintana and B. bacilliformis, in order to develop a universal treatment regimen active against all Bartonella-related infections.

\section{Materials and Methods}

\subsection{Bacterial strain, culture media and culture conditions}

The Bartonella henselae JK53 strain was obtained from BEI Resources (ATCC). B. henselae JK53 was cultured in Schneider's Drosophila medium (Life Technologies Limited, Paisley, UK) supplemented with 10\% fetal bovine serum (FBS) (Sigma-Aldrich, Co., St. Louis, MO, USA) and 5\% sucrose (Fisher Scientific, New Jersey, USA) in microaerophilic incubator without shaking at $37^{\circ} \mathrm{C}$, $5 \% \mathrm{CO}_{2}$. As previously measured [22], B. henselae JK53 rapidly went into logarithmic growth phase and reached a growth peak after 2 days under such culture conditions. The 1-day-old and 5-day-old culture were considered as log phase and stationary phase, respectively. The Columbia anaerobic sheep blood agar (HemoStat Laboratories, Dixon, CA, USA) was used to perform the drug exposure assay, which was also cultured at $37^{\circ} \mathrm{C}, 5 \% \mathrm{CO}_{2}$.

\subsection{Drugs, essential oils and their active ingredients}

A panel of 149 essential oils was purchased from Plant Therapy (ID, USA), Natural Acres (MO, USA), or Plant Guru (NJ, USA). Carvacrol and cinnamaldehyde were purchased from Sigma-Aldrich (USA). DMSO-soluble essential oils and carvacrol and cinnamaldehyde were dissolved in dimethyl sulfoxide (DMSO) at 5\% (v/v), followed by dilution at 1:10 into 5-day-old stationary bacteria cultures to achieve $0.5 \%$ final concentration. To make further dilutions for evaluating anti-Bartonella activity, the $0.5 \%$ essential oil treatments were further diluted in the same stationary bacteria cultures to achieve desired concentrations. DMSO-insoluble essential oils were added directly to 5-day-old stationary bacteria cultures to form aqueous suspension by adequate vortexing, followed by immediate transfer of the aqueous suspension into the same stationary cultures to make serial dilutions to achieve desired final concentrations. Antibiotics including azithromycin (AZI), daptomycin (DAP), doxycycline (DOX), gentamicin (GEN), methylene blue, miconazole, moxifloxacin (MXF), nitrofurantoin (NIT), rifampin (RIF) were purchased from Sigma-Aldrich and were dissolved in appropriate solvents [55] to form $10 \mathrm{mg} / \mathrm{mL}$ or $100 \mathrm{mM}$ stock solutions. All the antibiotic stocks were filter-sterilized by $0.2 \mu \mathrm{m}$ filter except the DMSO stocks and then diluted and stored at $-20^{\circ} \mathrm{C}$. 


\subsection{Microscopy techniques}

Drug-treated or control B. henselae JK53 cell suspensions were examined using BZ-X710 All-in-One fluorescence microscope (KEYENCE, Inc.), with SYBR Green I (100 × stock, Invitrogen) and propidium iodide (PI, $600 \mu \mathrm{M}$, Sigma) mixture used for staining. The SYBR Green I/PI dye was added to the sample at 1:10 dilution and mixed thoroughly to assess the bacterial viability by using the ratio of green/red fluorescence to determine the residual viability percentage, respectively, as described previously [18]. The residual bacteria viability was confirmed by analyzing three representative images of the same bacterial cell suspension using fluorescence microscopy. BZ-X Analyzer and Image Pro-Plus software were used to quantitatively determine the fluorescence intensity.

\subsection{Screening of essential oil library against stationary phase B. henselae JK53}

For the high-throughput essential oil screening, each essential oil was assayed in two concentrations, $0.5 \%(\mathrm{v} / \mathrm{v})$ and $0.25 \%(\mathrm{v} / \mathrm{v})$. Firstly, $20 \mu \mathrm{L} 5 \%$ essential oil DMSO stocks or aqueous suspension were added to the 96 -well plate containing $180 \mu \mathrm{L}$ of the 5-day-old stationary phase $B$. henselae JK53 culture to obtain the desired concentration of $0.5 \%$. And then the $0.25 \%$ treatment concentration was obtained by mixing $100 \mu \mathrm{L} 0.5 \%$ treatment with $100 \mu \mathrm{L}$ 5-day-old B. henselae JK53 culture. Antibiotics including AZI, DAP, DOX, GEN, methylene blue, miconazole, MXF, NIT, and RIF were used as control drugs at $20 \mu \mathrm{M}$. Plates were sealed and placed in $37^{\circ} \mathrm{C}$ incubator without shaking over of a period of 3 days. SYBR Green I/ PI viability assay was used to assess the live and dead cells after drug exposure as described [18]. Briefly, the SYBR Green I/PI dye was added to the sample at 1:10 dilution and mixed thoroughly. The plates were incubated at room temperature in the dark for 15 minutes followed by plate reading using microplate reader (HTS 7000 plus Bioassay Reader, PerkinElmer Inc., USA). The green/red $(538 \mathrm{~nm} / 650 \mathrm{~nm})$ fluorescence ratio of each well was used for calculating the residual viability percentage. With least-square fitting analysis, the regression equation and regression curve of the relationship between residual viability percentage and green/red fluorescence ratio was obtained, which was used in calculation as described previously [22]. All tests were run in triplicate.

\subsection{Drug exposure assay}

Based on primary screening results, active hits were further confirmed by drug exposure assay. The 5-day-old B. henselae JK53 stationary phase culture was used for drug exposure experiments, which was treated with $0.032 \%(\mathrm{v} / \mathrm{v})$ active essential oils respectively. Then two highly potent active ingredients, carvacrol and cinnamaldehyde, of active essential oils oregano and cinnamon bark, respectively, were also tested by drug exposure assay at a very low concentration of $0.01 \%(\mathrm{v} / \mathrm{v})$ and $0.005 \%(\mathrm{v} / \mathrm{v})$. Control antibiotics were used at their Cmax. The drug exposure assay was carried out in $15 \mathrm{~mL}$ Eppendorf tubes over the course of 7 days at $37^{\circ} \mathrm{C}, 5 \% \mathrm{CO}_{2}$ without shaking. At each time point we measured, the bacteria cells were collected by centrifugation and rinsed twice with fresh Schneider's medium followed by re-suspension in fresh Schneider's medium. Then the cell suspension was serially diluted and each dilution was plated on Columbia blood agar plates for viable bacterial counts (colony forming unit, CFU). All tests were run in triplicate.

\subsection{MIC determination}


The standard microdilution method was used to measure the minimum inhibitory concentration (MIC) needed to inhibit the visible growth of B. henselae JK53 after a 5-day incubation period. The diluted 1-day-old $B$. henselae JK53 logarithmic phase culture was used for MIC determination. $1 \times 10^{6}$ bacteria cells were inoculated into the well of the 96 -well plate containing 180 $\mu \mathrm{L}$ fresh modified Schneider's medium per well. Then $20 \mu \mathrm{L} 5 \%$ essential oil stocks were added into each well respectively to achieve $0.5 \%$ final concentration. Other lower concentrations were obtained by mixing $0.5 \%$ treatment with diluted 1-day-old $B$. henselae JK53 logarithmic phase culture. Plates were sealed and incubated at $37^{\circ} \mathrm{C}$ without shaking for 5 days. Then the bacteria cell proliferation was assessed using the SYBR Green I/PI assay and the bacterial counting chamber after the incubation. All tests were run in triplicate.

\section{Conclusions}

In summary, this is the first study of a high throughput drug screen against stationary phase $B$. henselae using a collection of essential oils where we identified a range of promising essential oils. Two active ingredients of these effective hits, carvacrol and cinnamaldehyde were also identified to have strong antimicrobial activity against stationary phase $B$. henselae, while other ingredients still need to be identified and evaluated thoroughly for both efficacy and toxicity. Future studies are needed to determine the activity of drug combinations with the identified essential oil candidates against Bartonella persisters as well as biofilm bacteria in vitro and in animal models of Bartonella infections. Our findings may have implications for developing improved treatment of persistent Bartonella infections in the future.

Author Contributions: Conceptualization, Y.Z.; Data curation, X.M.; Formal analysis, W.S.; Funding acquisition, Y.Z.; Writing - original draft, X.M.; Writing - review \& editing, Y.Z.

Funding: This research was funded by Steven \& Alexandra Cohen Foundation.

Acknowledgments: We acknowledge the support by Steven \& Alexandra Cohen Foundation. We thank BEI Resources (ATCC) for providing Bartonella henselae strain JK53 used in this study.

Conflicts of Interest: The authors declare no conflict of interest.

\section{References}

1. Brenner, D.J.; O'connor, S.P.; Winkler, H.H.; Steigerwalt, A.G. Proposals to unify the genera Bartonella and Rochalimaea, with descriptions of Bartonella quintana comb. nov., Bartonella vinsonii comb. nov., Bartonella henselae comb. nov., and Bartonella elizabethae comb. nov., and to remove the family Bartonellaceae from the order Rickettsiales. Int. J. Syst. Bacteriol. 1993, 43, 777-786.

2. Peters, D.; Wigand, R. Bartonellaceae. Bacteriol. Rev. 1955, 19, 150-159.

3. Okaro, U.; Addisu, A.; Casanas, B.; Anderson, B. Bartonella Species, an Emerging Cause of Blood-Culture-Negative Endocarditis. Clin. Microbiol. Rev. 2017, 30, 709-746.

4. Breitschwerdt, E.B. Bartonellosis, One Health and all creatures great and small. Vet. Dermatol. 2017, 28, 96-e21.

5. Maurya, A.K.; Mohanty, S.; Pal, A.; Chanotiya, C.S.; Bawankule, D.U. The essential oil from Citrus limetta Risso peels alleviates skin inflammation: In-vitro and in-vivo study. J. Ethnopharmacol. 2018, 212, 86-94.

6. Hong, J.; Li, Y.; Hua, X.; Bai, Y.; Wang, C.; Zhu, C.; Du, Y.; Yang, Z.; Yuan, C. Lymphatic Circulation Disseminates Bartonella Infection Into Bloodstream. J. Infect. Dis. 2017, 215, 303-311.

7. Rolain, J.M.; La Scola, B.; Liang, Z.; Davoust, B.; Raoult, D. Immunofluorescent detection of 
intraerythrocytic Bartonella henselae in naturally infected cats. J. Clin. Microbiol. 2001, 39, 2978-2980.

8. Jacomo, V.; Kelly, P.J.; Raoult, D. Natural history of Bartonella infections (an exception to Koch's postulate). Clin. Diagn. Lab. Immunol. 2002, 9, 8-18.

9. Chomel, B.B.; Boulouis, H.J.; Maruyama, S.; Breitschwerdt, E.B. Bartonella spp. in pets and effect on human health. Emerg. Infect. Dis. 2006, 12, 389-394.

10. Gutierrez, R.; Vayssier-Taussat, M.; Buffet, J.P.; Harrus, S. Guidelines for the Isolation, Molecular Detection, and Characterization of Bartonella Species. Vector. Borne. Zoonotic. Dis. 2017, 17, 42-50.

11. Angelakis, E.; Raoult, D. Pathogenicity and treatment of Bartonella infections. Int. J. Antimicrob. Agents. 2014, 44, 16-25.

12. Biswas, S.; Rolain, J.M. Bartonella infection: treatment and drug resistance. Future. Microbiol. 2010, 5, 1719-1731.

13. Zhang, Y. Persisters, persistent infections and the Yin-Yang model. Emerg. Microbes. Infect. 2014, 3, e3.

14. Lee, M.S.; Choi, J.; Posadzki, P.; Ernst, E. Aromatherapy for health care: an overview of systematic reviews. Maturitas. 2012, 71, 257-260.

15. Hyldgaard, M.; Mygind, T.; Meyer, R.L. Essential oils in food preservation: mode of action, synergies, and interactions with food matrix components. Front. Microbiol. 2012, 3, 12.

16. Singh, G.; Kapoor, I.P.; Pandey, S.K.; Singh, U.K.; Singh, R.K. Studies on essential oils: part 10; antibacterial activity of volatile oils of some spices. Phytother. Res. 2002, 16, 680-682.

17. Sakkas, H.; Gousia, P.; Economou, V.; Sakkas, V.; Petsios, S.; Papadopoulou, C. In vitro antimicrobial activity of five essential oils on multidrug resistant Gram-negative clinical isolates. J. Intercult. Ethnopharmacol. 2016, 5, 212-218.

18. Feng, J.; Wang, T.; Zhang, S.; Shi, W.; Zhang, Y. An optimized SYBR Green I/PI assay for rapid viability assessment and antibiotic susceptibility testing for Borrelia burgdorferi. PLoS One. 2014, 9, e111809.

19. Feng, J.; Zhang, S.; Shi, W.; Zubcevik, N.; Miklossy, J.; Zhang, Y. Selective Essential Oils from Spice or Culinary Herbs Have High Activity against Stationary Phase and Biofilm Borrelia burgdorferi. Front. Med (Lausanne). 2017, 4, 169.

20. Feng, J.; Shi, W.; Miklossy, J.; Zhang, Y. Additional Essential Oils with High Activity against Stationary Phase Borrelia burgdorferi. Bioarxiv [preprint]. 2018, 260091.

21. Feng, J.; Shi, W.; Zhang, S.; Sullivan, D.; Auwaerter, P.G.; Zhang, Y. A Drug Combination Screen Identifies Drugs Active against Amoxicillin-Induced Round Bodies of In Vitro Borrelia burgdorferi Persisters from an FDA Drug Library. Front. Microbiol. 2016, 7, 743.

22. Li, T.; Feng, J.; Xiao, S.; Shi, W.; Sullivan, D.; Zhang, Y. Identification of FDA-Approved Drugs with Activity against Stationary Phase Bartonella henselae. Antibiotics (Basel). 2019, 8.

23. Dörbecker, C.; Sander, A.; Oberle, K.; Schuilin-Casonato, T. In vitro susceptibility of Bartonella species to 17 antimicrobial compounds: Comparison of Etest and agar dilution. J. Antimicrob. Chemother. 2006, 58, 784-788.

24. Rolain, J.M.; Brouqui, P.; Koehler, J.E.; Maguina, C.; Dolan, M.J.; Raoult, D. Recommendations for treatment of human infections caused by Bartonella species. Antimicrob. Agents. Chemother. 2004, 48, 1921-1933.

25. Feng, J.; Wang, T.; Shi, W.; Zhang, S.; Sullivan, D.; Auwaerter, P.G.; Zhang, Y. Identification of novel activity against Borrelia burgdorferi persisters using an FDA approved drug library. Emerg. Microbes. Infect. 2014, 3, e49.

26. Ben Hsouna, A.; Ben Halima, N.; Smaoui, S.; Hamdi, N. Citrus lemon essential oil: chemical composition, 
antioxidant and antimicrobial activities with its preservative effect against Listeria monocytogenes inoculated in minced beef meat. Lipids. Health. Dis. 2017, 16, 146.

27. Aboelhadid, S.M.; Mahrous, L.N.; Hashem, S.A.; Abdel-Kafy, E.M.; Miller, R.J. In vitro and in vivo effect of Citrus limon essential oil against sarcoptic mange in rabbits. Parasitol. Res. 2016, 115, 3013-3020.

28. Maurya, A.K.; Mohanty, S.; Pal, A.; Chanotiya, C.S.; Bawankule, D.U. The essential oil from Citrus limetta Risso peels alleviates skin inflammation: In-vitro and in-vivo study. J. Ethnopharmacol. 2018, 212, 86-94.

29. Shen, C.Y.; Jiang, J.G.; Zhu, W.; Ou-Yang, Q. Anti-inflammatory Effect of Essential Oil from Citrus aurantium L. var. amara Engl. J. Agric. Food. Chem. 2017, 65, 8586-8594.

30. Siti, H.N.; Kamisah, Y.; Nur Iliyani, M.I.; Mohamed, S.; Jaarin, K. Citrus leaf extract reduces blood pressure and vascular damage in repeatedly heated palm oil diet-Induced hypertensive rats. Biomed. Pharmacother. 2017, 87, 451-460.

31. Bejaoui, A.; Chaabane, H.; Jemli, M.; Boulila, A.; Boussaid, M. Essential oil composition and antibacterial activity of Origanum vulgare subsp. glandulosum Desf. at different phenological stages. J. Med. Food. 2013, 16, 1115-1120.

32. Shelef L.A.; Naglik O.A.; Bogen D.W. Sensitivity of some food-borne to the spices sage, rosemary and allspice. J. Food. Sci. 1980, 45, M1042-1044.

33. Eskow, E.; Rao, R.V.; Mordechai, E. Concurrent infection of the central nervous system by Borrelia burgdorferi and Bartonella henselae: evidence for a novel tick-borne disease complex. Arch. Neurol. 2001, 58, 1357-1363.

34. Ren, P.; Ren, X.; Cheng, L.; Xu, L. Frankincense, pine needle and geranium essential oils suppress tumor progression through the regulation of the AMPK/mTOR pathway in breast cancer. Oncol. Rep. 2018, 39, 129-137.

35. Lysakowska, M.E.; Sienkiewicz, M.; Banaszek, K.; Sokolowski, J. The Sensitivity of Endodontic Enterococcus spp. Strains to Geranium Essential Oil. Molecules. 2015, 20, 22881-22889.

36. Al-Yasiry, A.R.; Kiczorowska, B. Frankincense--therapeutic properties. Postepy. Hig. Med. Dosw (Online). 2016, 70, 380-391.

37. Ndoti-Nembe, A.; Vu, K.D.; Han, J.; Doucet, N.; Lacroix, M. Antimicrobial Effects of Nisin, Essential Oil, and gamma-Irradiation Treatments against High Load of Salmonella typhimurium on Mini-carrots. J. Food. Sci. 2015, 80, M1544-1548.

38. Sue, C.; Gary, Y.; Craig, O.; Karen, N.; Inhibition of methicillin-resistant Staphylococcus aureus (MRSA) by essential oils. Flavour Fragr. J. 2008, 23, 444-449.

39. Ndoti-Nembe, A.; Vu, K.D.; Doucet, N.; Lacroix, M. Antimicrobial effects of essential oils, nisin, and irradiation treatments against Listeria monocytogenes on ready-to-eat carrots. J. Food. Sci. 2015, 80, M795-799.

40. Broznic, D.; Ratkaj, I.; Malenica Staver, M.; Kraljevic Pavelic, S.; Zurga, P.; Bubalo, D.; Gobin, I. Evaluation of the Antioxidant Capacity, Antimicrobial and Antiproliferative Potential of Fir (Abies alba Mill.) Honeydew Honey Collected from Gorski kotar (Croatia). Food. Technol. Biotechnol. 2018, 56, 533-545.

41. Gobin, I.; Crnkovic, G.; Magdalenic, M.; Begic, G.; Babic, A.; Lusic, D.; Vuckovic, D. Antibacterial potential of Croatian honey against antibiotic resistant pathogenic bacteria. Med. Glas (Zenica). 2018, 15, 139-144.

42. Whiley, H.; Gaskin, S.; Schroder, T.; Ross, K. Antifungal properties of essential oils for improvement of indoor air quality: a review. Rev. Environ. Health. 2018, 33, 63-76.

43. Tan, L.T.; Lee, L.H.; Yin, W.F.; Chan, C.K.; Abdul Kadir, H.; Chan, K.G.; Goh, B.H. Traditional Uses, Phytochemistry, and Bioactivities of Cananga odorata (Ylang-Ylang). Evid. Based. Complement. Alternat. Med. 
2015, 2015, 896314.

44. Almeida Lde, F.; Paula, J.F.; Almeida, R.V.; Williams, D.W.; Hebling, J.; Cavalcanti, Y.W. Efficacy of citronella and cinnamon essential oils on Candida albicans biofilms. Acta. Odontol. Scand. 2016, 74, 393-398.

45. Oliveira, J.B.; Teixeira, M.A.; Paiva, L.F.; Oliveira, R.F.; Mendonca, A.; Brito, M.J.A. In Vitro and In Vivo Antimicrobial Activity of Cymbopogon citratus (DC.) Stapf. Against Staphylococcus spp. Isolated from Newborn Babies in an Intensive Care Unit. Microb. Drug. Resist. 2019.

46. Mogana, R.; Teng-Jin, K.; Wiart, C. In Vitro Antimicrobial, Antioxidant Activities and Phytochemical Analysis of Canarium patentinervium Miq. from Malaysia. Biotechnol. Res. Int. 2011, 2011, 768673.

47. David, A.; Wang, F.; Sun, X.; Li, H.; Lin, J.; Li, P.; Deng, G. Chemical Composition, Antioxidant, and Antimicrobial Activities of Vetiveria zizanioides (L.) Nash Essential Oil Extracted by Carbon Dioxide Expanded Ethanol. Molecules. 2019, 24.

48. Powers, C.N.; Osier, J.L.; Mcfeeters, R.L.; Brazell, C.B.; Olsen, E.L.; Moriarity, D.M.; Satyal, P.; Setzer, W.N. Antifungal and Cytotoxic Activities of Sixty Commercially-Available Essential Oils. Molecules. 2018, 23.

49. Zouhir, A.; Jridi, T.; Nefzi, A.; Ben Hamida, J.; Sebei, K. Inhibition of methicillin-resistant Staphylococcus aureus (MRSA) by antimicrobial peptides (AMPs) and plant essential oils. Pharm. Biol. 2016, 54, 3136-3150.

50. Gadisa, E.; Weldearegay, G.; Desta, K.; Tsegaye, G.; Hailu, S.; Jote, K.; Takele, A. Combined antibacterial effect of essential oils from three most commonly used Ethiopian traditional medicinal plants on multidrug resistant bacteria. BMC. Complement. Altern. Med. 2019, 19, 24.

51. Orchard, A.; Van Vuuren, S. F.; Viljoen, A.M.; Kamatou, G. The in vitro antimicrobial evaluation of commercial essential oils and their combinations against acne. Inter. J. Cosmet. Sci, 2018, 40, 226-243.

52. Lanzerstorfer, A.; Hackl, M.; Schlomer, M.; Rest, B.; Deutsch-Grasl, E.; Lanzerstorfer, C. The influence of air-dispersed essential oils from lemon (Citrus limon) and silver fir (Abies alba) on airborne bacteria and fungi in hospital rooms. J. Environ. Sci. Health. A. Tox. Hazard. Subst. Environ. Eng. 2019, 54, 256-260.

53. Breitschwerdt, E.B.; Kordick, D.L. Bartonella infection in animals: carriership, reservoir potential, pathogenicity, and zoonotic potential for human infection. Clin. Microbiol. Rev. 2000, 13, 428-438.

54. Pulliainen, A.T.; Dehio, C. Persistence of Bartonella spp. stealth pathogens: from subclinical infections to vasoproliferative tumor formation. FEMS. Microbiol. Rev. 2012, 36, 563-599.

55. Performance Standards for Antimicrobial Susceptibility Testing. Available online: https://clsi.org/standards/products/microbiology/documents/m100/(accessed on 13 December 2018) 